\section{THE TREATMENT OF WAR WOUNDS.?}

$W^{E}$ are wont to classify the patients in our military hospitals into sick and wounded. In reality all, or nearly all, are suffering from bacterial infections. And the essential difference between the sick and the wounded lies in this, that the sick are suffering from infections spontaneously contracted, the wounded from infections induced by mechanical injuries. My theme is the treatment of this latter class of infections. They are distinguished by certain quite special features.

In spontaneous infection we have to deal with microbes which have fought their way into the body, and generally only a single species of microbe will have done this. In wounds we have nicrobes mechanically driven in, and every sort of microbe which exists in external Nature may thus be introduced.

But let me, before embarking upon the question of their treatment, first tell you something about the

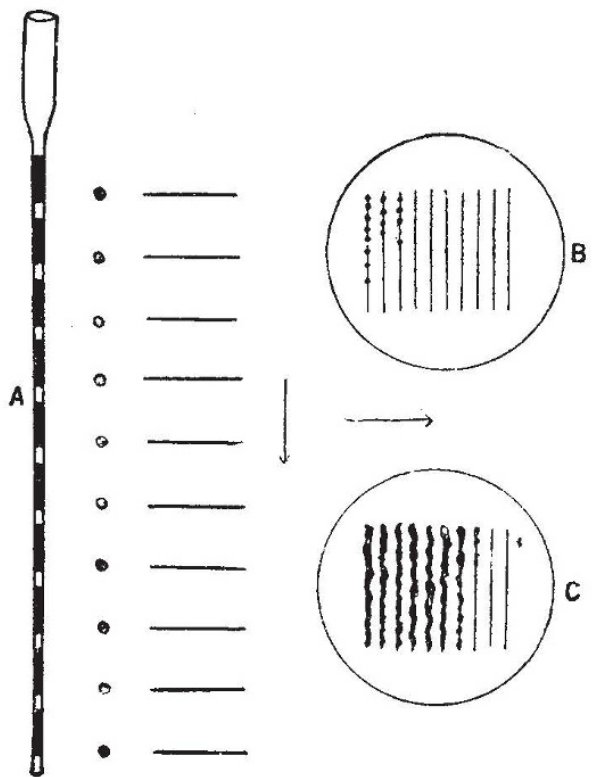

FIG. I. - Method of pyo-sero-culture. A, Pipette which has been implanted by the wet-wall metrod, and has then been filled in by the wash and after-wash procedure with unit-volumes of serum. By the side of the pipetie to the right is ranged a series of drops representing the series of unit-volumes of serum blown out in order from the pipette, and, finally, to the right of the drops is a series of lines representing linear implantations made upon agar. B, Results of the series of linear implantations made wich the unit-volumes of the patient's serum. C, Results of the series of linear implantations made with the unit volumes of the normal serum which was used as a control.

natural agencies by which the inroads of microbes are combated. You are, of course, aware that we are guarded against microbic infection by our blood fluids and our white blood corpuscles.

\section{The Body Fluids.}

Let me begin with the blood fluids, and let me take you directly to the following experiment. I call it the experiment of pyo-sero-culture-i.e. the experiment in which we implant pus into serum to see which of the microbes of the wound can grow in the blood fluids.

We procure for our experiment a suppurating wound. We take from it a specimen of pus containing a large variety of different organisms. At the same time we take from the patient's finger a sample

1 By Sir Almroth E. Wright, C.B., F.R.S. In its original form this lecture was delivered at the Koyal Institution on March 9. It was supplemented by additional matter relating to antiseptics and the method of Carrel, and was printed in full in the Lancet of June 23. Parts of the lecture of purely technical interest have been omitted. of blood; and we take a specimen also of our own. When the serum has issued from the clot we take a capillary pipette, fit a rubber teat to the barrel, and inscribe a mark upon the stem at about, say, one-third of an inch from the tip. We now aspirate a little pus into the stem, drawing it up only so far as our fiducial mark, and, blowing it out again, leave a wash of pus upon the walls. This done, we sterilise the tip of the pipette, and then aspirate into the stem a series of unitvolumes of serum, dividing each volume off from the next by a bubble of air. The pipette when filled in this manner presents the appearance shown in Fig. I, and we have in the proximal end our first and heaviest implantation of pus, and in the distal end our last and lightest implantation. The pipette is now placed in the incubator to allow every microbe which is capable of growing in serum to do so. After an interval of six or more hours we proceed to our examination. What we do is to blow out our series of unit-volumes of serum in separate drops and examine under the microscope; or, better, we plant out a sample of each drop upon a separate seed-bed. Here in B and $C$ you have the results of such culture represented diagrammaticallythe meagre crop in $B$ being that obtained with the patient's serum, and the more copious crop in $\mathrm{C}$ being that obtained with normal serum.

And you have in the next figure (Fig. 2) a drawing of an agar tube implanted from a pyo-sero-culture made with the serum of a wounded man. In the upper part of the agar tube you see two seed-plots implanted from the distal portion of the capillary stem. These have remained sterile. In the middle of the tube you see four plots implanted from the unit-volumes of serum which occupied the middle region of the capillary stem. These have grown colonies of only one species of microbe-the streptococcus. At the bottom of the tube you see seed-plots implanted from the proximal end of the capillary stem. These are overgrown with colonies of staphylococcus. But no doubt interspersed with, and overgrown by, these are also colonies of streptococci. If, instead of cultures from the patient's serum, I had been showing you here cultures from normal serum, what you would have seen would have been a much larger number of fertile seed-plots, and the seed-plots implanted from the

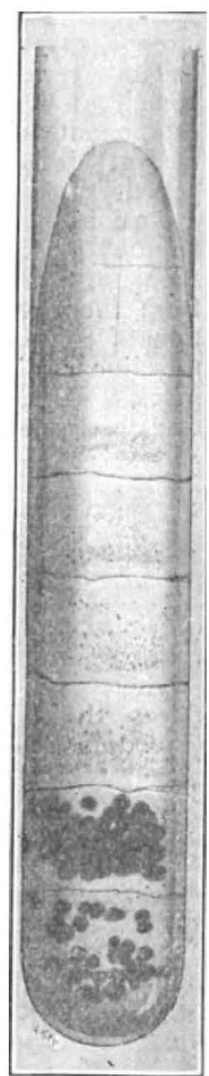

FI(i. 2. $_{\text {- A portion of }}$ a pyo-sero-culture planted out upon an agar slant divided up by furrows into a series of seed-beds. proximal end of the pipette would have shown a large assortment of different colonies.

We learn from such experiments three lessons: first, that in the uncorrupted serum in the distal region of the pipette only two species of microbes from the wound can grow and multiply; secondly, that in the corrupted serum in the proximal end of the pipette all the microbes of the wound can grow; and, thirdly, we learn from a comparison of the wounded man's serum with the normal serum that the former offers more resistance to microbic srowth, and is less easily corrupted by the addition of pus.

Cause of the Corruption of the Serum.

Experiments of this kind clearly do not tell us the cause of the corruption of the serum. That corruption 
may be due to some chemical substance contributed by the pus to the serum or to something special in the character of the bacteria implanted. This point we can clear up as follows. We go back to our very septic wound. We clean it out carefully by syringing. That leaves us with a wound cavity clean but still abundantly infected. We then take the little cupping apparatus which is shown in Fig. 3. We apply it to the walls of the wound, using light pressure. Then, puncturing the attached rubber tube with the needle of a hypodermic syringe, we withdraw the contained air, and leave our lymph leech in situ adhering by negative pressure until the time for redressing the wound comes round. When we now go back to our wound we find there two quite different discharges. We have in the general cavity of the wound a thick pus containing many broken-down leucocytes and pullulating with all sorts of microbes. In the body of the lymph leech we have a nearly clear lymph containing well-preserved leucocytes and only a very few staphylococci and streptococci. Since we had on every part of the walls precisely the same amount and kind of bacterial infection, and since we are in each case dealing with the self-same lymph and leucocytes, this difference of results is imputable, not to our having in the lymph leech a different bacterial implantation, but to the negative pressure having furnished a larger proportion of blood fluids.

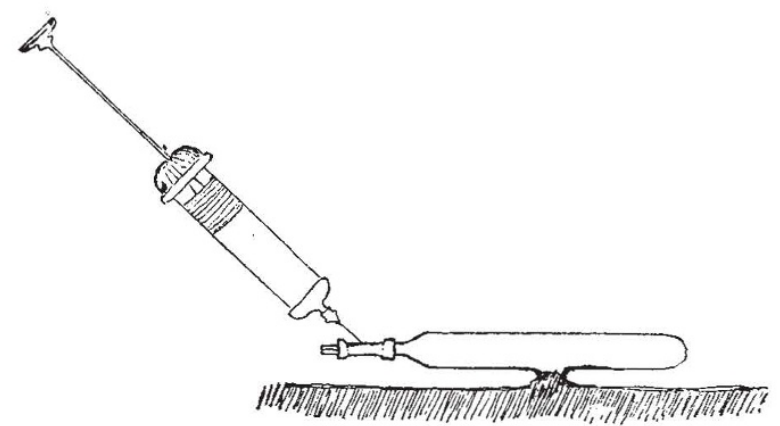

Fig. 3.-Lymph leech in position, showing technique for exhausting the air.

But with this the problem is, as you see, only incompletely resolved. We have learned that the corruption of the lymph is not determined by the nature of the bacterial implantation; we have reason to think it is hindered by a larger afflux of lymph; and it looks as if it might have something to do with the breaking down of the leucocytes. But we have not yet put our finger upon the particular element that takes away from the serum its power of inhibiting microbic growth, and converts it into a congenial pabulum for all manner of micro-organisms.

Let me in this connection invite you to consider-for that may perhaps put us on the path for the solution of our problem-a scheme of classification of the albuminous substances. I would propose to classify them from the point of view of their capacity to furnish pabulum for microbes, and to distinguish three classes of albuminous sulstances. First would come digested albumens. It is familiar matter that these furnish very congenial pabulum for microbes. In the form of peptone we use them for all our artificial cultures. A second category of albumens would be native albumens. Muscle, milk, and eggs furnish such albumens. These are not like digested albumens, directly assimilable. Before they can be assimilated, whether by ourselves or by microbes, they must be broken down into simpler elements by digestion. To that end we, and a certain number of microbes also, are furnished with digestive ferments. There is yet a third class of albu- mens. I would venture to call these defended or pro. tected albumens. These cannot, like the digested albumens, be directly assimilated. Nor can they, like the native albumens, be directly digested. They are specially defended against the attack of digestive ferments. The albumens of the serum fall into this class of "defended albumens." It is well known with respect to serum that it has an antizymotic, and in particular an antitryptic, power-a power of neutralising digestive ferments, and in particular trypsin. You will, perhaps, not immediately perceive that the fact that the serum is antitryptic in any way elucidates our problem. But let us take that fact and put it in another way and then consider. Let us, instead of saying that the serum has an antitryptic property, say that it has a power of preventing its constituent albumens being converted into pabulum for microbes, and immediately, as I think, light is projected upon our problem. For once we envisage the facts in that way we are immediately impelled to inquire whether the serum's power of inhibiting bacterial growth may not be due to its power of neutralising digestive ferments, and whether the corruption of the lymph in the cavity of the wound may not be due to a collapse of its defence against proteolytic attack.

That is a point which is very easily settled by direct experiment. And let me now show you what happens when we add trypsin to a serum which has been implanted with microbes. I have here two tubes of a serum implanted two days ago with a minute quantity of pus containing a variety of different microbes. To the one I added trypsin, the quantity added being less than that required to neutralise its antitryptic power. The other tube of the implanted serum served as a control. Both tubes were then placed in the incubator. And you see the difference. The trypsinised serum is turbid with microbic growth. That is, we have here exactly the same result as that obtained in our pyosero-culture in those volumes of serum which were corrupted by a heavy implantation of pus; and the same result also as was in the lymph leech experiment obtained in the discharges in the wound cavity. Our control serum has, as you see, remained almost perfectly clear. That is exactly the same result as was obtained in our pyo-sero-culture in the distal end of our tube, and again in our lymph leech experiment in the cavity of the lymph leech.

And the doctrine that the antitryptic power is the protector, and trypsin the corrupter, of the blood fluids wins further support from the following facts:--(I) In every suppurating wound there is, as we shall presently see, a source from which trypsin can be derived. (2) Blood fluids which inhibit microbic growth are strongly antitryptic; and blood fluids which we find teeming with microbes are tryptic. (3) Examination of the blood shows that all wounded men have a markedly increased antitryptic power, and heavily wounded men (vou saw in our pyo-sero-culture what results from this) on an average a three- or four-fold increased antitryptic power. That clearly teaches that the body when endangered takes steps to protect itself nonspecifically against all microbic infections of the blood fluids.

\section{The Leucocytes.}

I now pass on to consider the leucocytes and the part they play in the destruction of microbes. You already know with respect to leucocytes that they can emerge from the blood-vessels, burrowing their way out through small pores in the capillary walls; that they make their way to every focus of infection; that they ingest microbes when these have first been prepared by the action of the blood fluids; and, finally, that they can, if things go favourably, digest and dissolve the ingested microbes. There would by consequence in connection with the leucocvte be 
three functions to study. First would come emigration, then phagocytosis, and lastly intracellular digestion. Emigration has up to the present been studied only in the interior of the organism You will realise that means that it has been studied only in a difficult setting and in the presence of all manner of disturbing factors, and you will appreciate that we want now a new and better technique. For we require for the treatment of the infected wound to find out how best to call out the leucocytes; and how, when occasion requires, to restrain their emigration.

I have in connection with this a technique to describe to you; but first I want you to appreciate what we can and what we cannot expect from leucocytes in the matter of locomotion. Leucocytes can, we know, make their way out through small openings. They can also travel over any ordinary surface. They can edge their way along faster when lightly compressed between two surfaces. They can crawl along strands, creep through a meshwork, and climb a scaffolding. But they are unable to climb a vertical glass wall. And again, they are unable to swim, and so once they get into open fluid they simply go to the bottom. We may liken them to very minute slugs crawling along surfaces and climbing trellises, but brought up short by any considerable barrier of fluid.

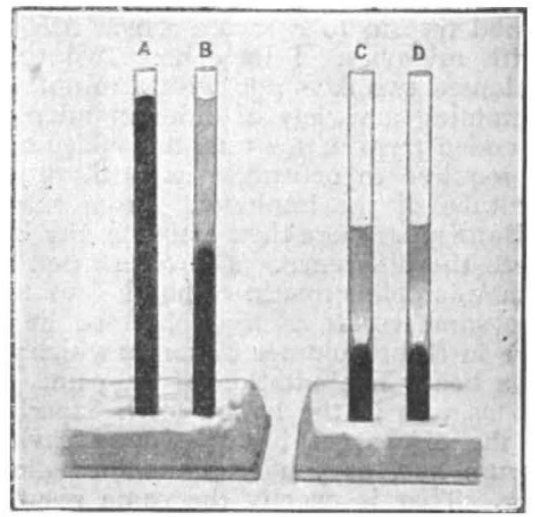

FIG. 4.--Drawing of four flattened capillary tubes. A, filled in with blood ; B, a similar tube after centrifugalisation showing above the "white" and below the "red clot"; $\mathrm{C}$ and $\mathrm{D}$, similar tubes after incubation. Leucocytic emigration is in each case visible to the naked eye as an opaque white band occupying the lower portion of the white clot. In $\mathrm{D}$, where physiological salt solution had been imposed upon the white clot, the band of emigration is much broader than in $C$.

All these points must be considered when seeking for a technique for the experimental study of emigration, using for that study specimens of blood withdrawn from the body. The containing blood-vessel can up to a point be imitated by a glass tube, and we can, to facilitate observation, use tubes drawn out flat, such as shown in Fig. 4. But the artificial differs from our natural capillary in having impermeable instead of permeable walls. This, of course, makes emigration through the walls impossible. None the less, these tubes supply what we want for the study of the movements of leucocytes. We can institute races along the length.

But first certain preparations must be made. The course nust be cleared of all obstructions-i.e. the red corpuscles must be got out of the way. Next the leucocytes must all be brought back behind the scratch line. Further, we must provide a scaffolding for the leucocytes to climb. All this can be arranged. We fill in our flat emigration tubes with blood and seal them at one end. Then, by centrifuging, we bring the blood fluids to the top and the corpuscles to the bottom. The lighter leucocytes will now have arranged themselves in a layer immediately above the red; and presently the supernatant fluid will clot and the meshwork of fibrin will then provide the scaffolding we require. We can now impose upon the clot-let me for convenience call it the white clot--any chemical agent we please and let it slowly diffuse down to the leucocytes. For the study of the effect of bacterial infection, we can introduce microbes into the blood before this is filled into the tube. Or, as an alternative, blood can be filled into tubes the walls of which have been wetted with a microbic culture. Finally, we set our experiment going by placing our emigration tubes in the incubator-that is, we supply to our leucocytes the necessary warmth.

And we can at any moment take stock of what is occurring in our tubes by examining through the walls with the naked eye or with the low power of the microscope. Also, by a very simple technique we can extract the clot from the tube and mount and colour it, so as to bring everything clearly into view under the high powers of the microscope.

Emigration of Leucocytes: Facts with Practical Application.

I must limit myself to showing you in connection with emigration a few outstanding facts which have a practical application to the treatment of wounds. Let me begin with the naked-eye appearances. We have in Fig. 4, C and D, emigration $t u b e s$ containing centrifuged blood which has been in the incubator for about eight hours. In $\mathrm{C}$ - the control tube

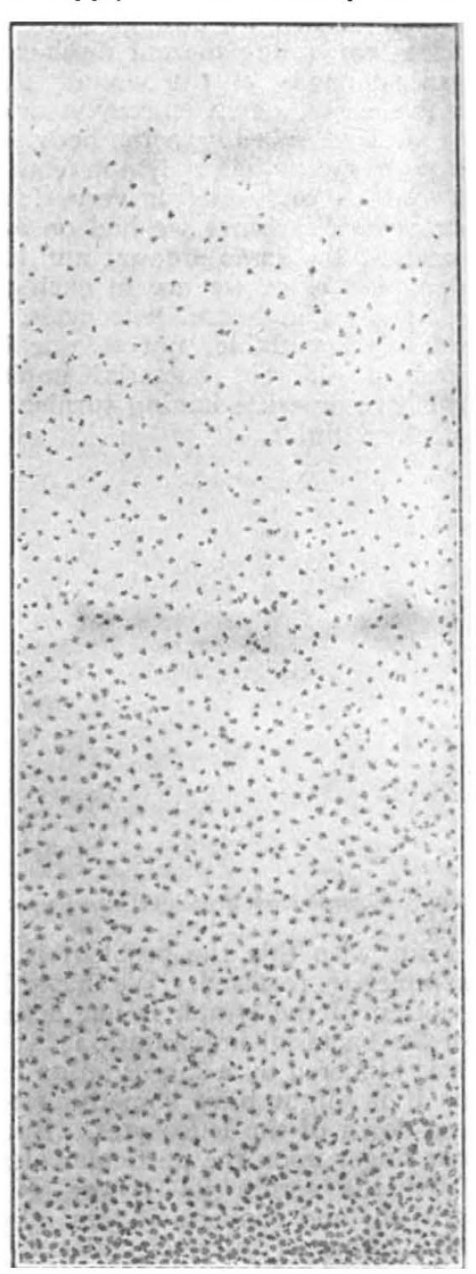

FIG. 5.-Magnified view of the band of leucocytic emigration seen in Fig. $4, D$. -we have centrifuged blood to which no addition has been made. In $\mathrm{D}$ some weak salt solution has been imposed upon the white clot. The emigrating leucocytes are visible to the eye in the form of a slightly opaque white band extending upwards from the red into the white clot. You see that in D the corpuscles have climbed higher than in $\mathrm{C}$.

Fig. 5 shows what such a band of emigrating leucocytes looks like under the microscope. Instead of the leucocytes being all, as you will see in the next figure, congregated together behind the starting line, they here are actively emigrating-the more active outdistancing the others in the race.

Fig. 6 shows what happens when 5 per cent. salt solution is imposed upon the blood. That salt passes

No. 2495, VOL. 99] 
down by diffusion and arrests emigration, and I wart you to notice on the right of the figure (and more clearly in the inset) that the few white corpuscles which were beginning to emigrate when the salt solution overtook them are broken up and destroyed. By that trypsin will be set free.

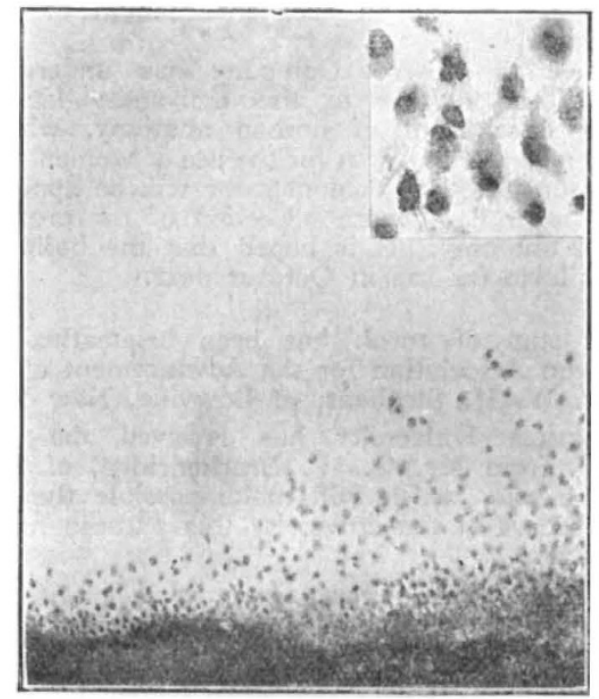

FIG. 6.-Magnified view of the leucocytic layer in the case where strong salt solution was superposed upon the

I next show you what happens when microbes have been implanted into the blood. Those microbes-supposing always that they are the sort that can proliferate in blood-grow out into colonies. In Fig. 7 is shown what happens when an excessive implantation has been made, and the bacterial colonies come up very

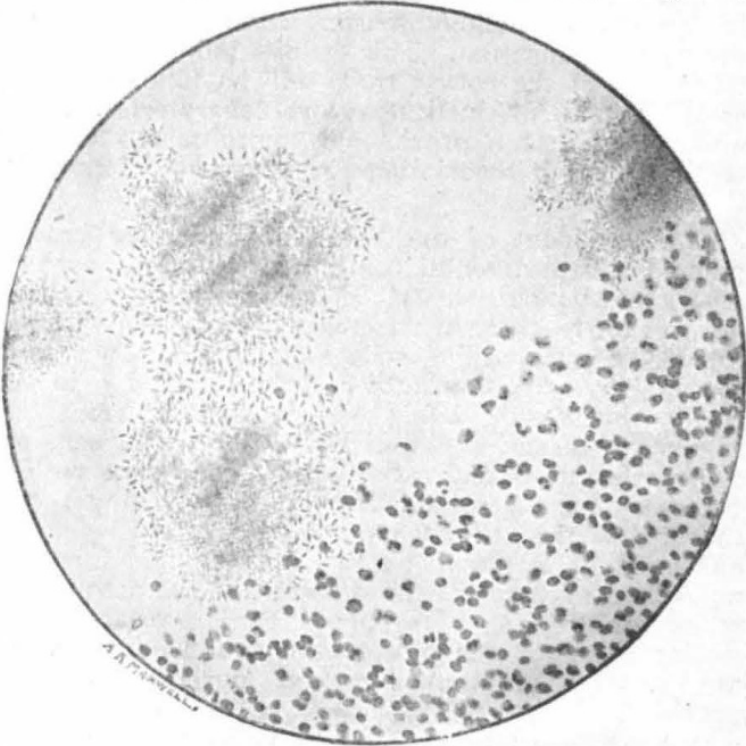

FIG. 7.-Leucocytic emigration restrained by excess of streptococcic infection.

thickly in the blood. You see here that emigration is entirely arrested. If that were to happen in infected tissues it would mean that the organism was there giving up the combat against the microbes.

In Fig. 8 we have again streptococcus implanted into the blood, but this time it is a much more sparing implantation. And here, as you see, the leucocytes are carrying out a raid against the microbes, each leucocyte ingesting and filling itself full with microbes.

In Fig. 9 I show you what happens when we make into the blood a very heavy implantation of the gangrene bacillus. Here in the neighbourhood of the leucocytic

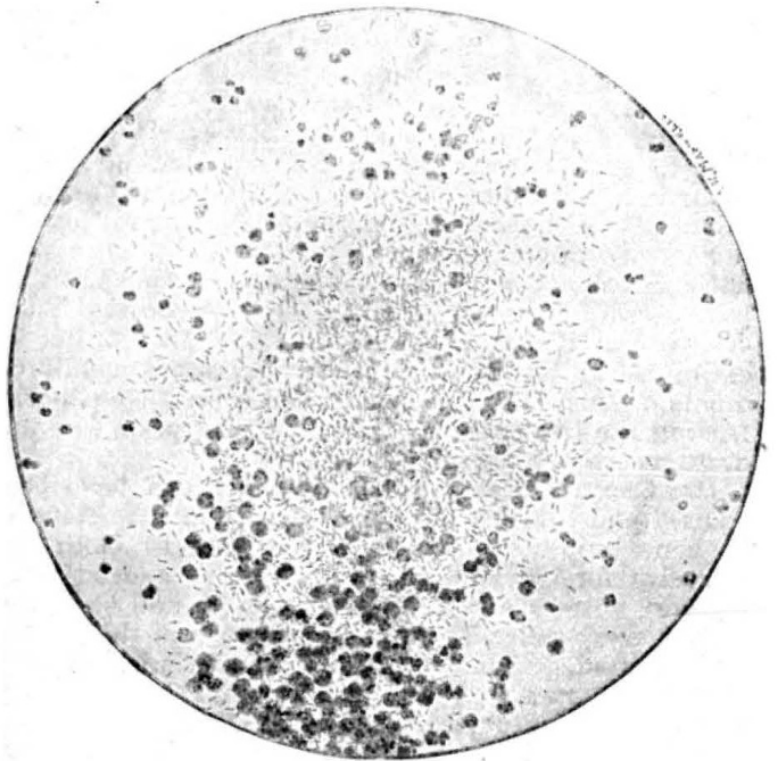

FIG. 8.-Leucocytes emigrating and attacking a colony of streptococci.

layer things are for the moment going well with the leucocytes, for they are actively phagocyting. But farther away from that layer there are very numerous colonies of the gangrene bacillus, which are growing unimpeded. The omens are consequently unfavourable. You can see in your mind's eye what is going to

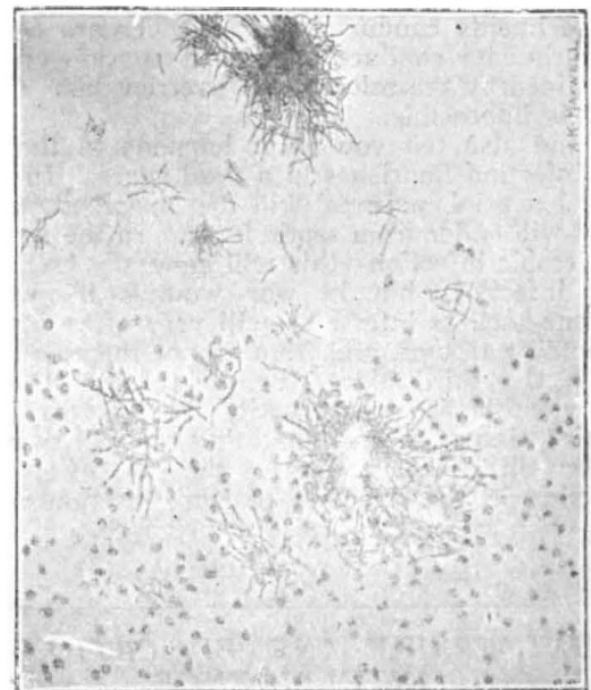

FIG. 9.-Leucocytes emigrating and attacking colonies of the gas gangrene bacillus.

happen. In the first place, all further emigration of leucocytes is going to be ariested; and, in the second, the leucocytes which have already emigrated and ingested microbes will, instead of successfully digesting them, be gradually poisoned by bacterial toxins. And when the leucocytes are killed, their 
digestive ferment-trypsin-passes out into the serum. By that, the serum will, as we have seen, be converted into a medium in which microbes can grow and pullulate.

Distinction between "Live" and "Dead" Spacis.

But we must now come back from these general questions to that of the treatment of the wound. Let me begin by explaining to you-for important questions of treatment hinge upon this-the distinction betiveen "live spaces" and "dead spaces."

In the lacunæ of vascularised tissues we have live spaces. In these we have optimum conditions for resistance to bacterial infection. We have here an antibacterial lymph; and by continuously renewed exudation corruptive changes will be continuousily antagonised and made good. Again, in live spaces we have terrain that can be effectively searched by leucocytes; and if the bacterial infection should not be extinguished by the first leucocytic attack reinforcements of leucocytes can be supplied from the capillaries feeding the live spaces. In all these respects live and dead spaces are sharply contrasted.

Dead spaces are found in tissues which have been bruised and cut off from their blood-supply, in sloughs. in bone sequestra, and in the texture of cloth and intrusive foreign bodies. And we have a dead space in every abscess sac under every slough and scab, and also in every collection of pus lying open in the pockets of a wound. The essential characteristics of deac spaces are two. First, they cannot be effectively searched by leucocytes. In some cases chemical, in others mechanical, conditions stand in the way. Secondly, the leucocytes which are engaged cannot be reinforced, nor can the fluid be renewed. Isolation from the circulation makes this impossible.

Let me try to tell you in the fewest possible words what this imports in infection. If in an infected live space the scale turns in favour of the microbes, there is still a chance of the balance being redressed. At any rate, reinforcements can be brought up. In infected dead spaces-and let me here point out to you that all experiments conducted in test-tubes are equivalent to experiments conducted in dead spaces-an advantage gained by the microbes is irretrievable. Counterattack is impossible.

Let me also tell you what happens to the patient when infection flourishes in a dead space. In the first place, bacterial poisons will be absorbed, and the patient will suffer from septic fever. In the next place, the microbic infection-this will generally be a streptococcic infection, but in war wounds it may be a gangrene-bacillus infection-will very often invade the surrounding tissues, and from thence the blood-strean. And in the third place, the pus in the dead space will, when it becomes tryptic, eat its way into the enclosing tissues. The containing sac will then extend in everv direction, the pus in the case of an abscess burrowing in the direction of least resistance until it opens upon an inner or an outer surface.

(To be continued.)

\section{UNIVERSITY AND EDUCATIONAL INTELLIGENCE.}

Oxfond.-By the death of Arthur Cornwallis Madan, senior student of Christ Church, the University loses one who was not only an accomplished scholar in the usual sense, but also perhaps the chief living authority on the native languages of Central Africa. His work in connection with the Universities' Mission was carried on for many years both at Zanzibar and in the tropical interior, and resulted in the accumulation and arrangement of a large mass of valuable material, both philological and grammatical. Mr. Madan was the son of a Canon of Gloucester; one of his brothers is the learned and energetic Librarian of the Bodleian, and another (the late H. G. Madan), a fellow of Queen's, was for many years science master at Eton, and was well known as joint author with Mr. A. G. Vernon Har. court, F.R.S., of an excellent manual of practical chemistry.

The Clothworkers' Company has undertaken to provide an addition to the University laboratories in the department of human anatomy, which will supply a dissecting-room for the use of women students. A woman assistant demonstrator will be appointed to teach under the genera direction of the professor of human anatomy. It is hoped that the building will be available for use in October next.

THE sum of roool. has been bequeathed to the American Association for the Advancement of Science by Mr. W. H. Stephens, of Lowville, New York.

Colcmbia University has received the sum of ro,oool. from Mr. G. W. Brackenridge, of San Antonio, Texas, which will make possible the opening of the doors of the university this autumn to women students.

AN educational campaign against tuberculosis throughout the Army at home and abroad has been undertaken by the National Council of the Y.M.C.A The campaign will be conducted by means of information imparted in the huts of the association. Dr. H. Sutherland will inaugurate the work by giving an address on "Consumption: Its Causes and Cure," at the Central Institute of the Y.M.C.A., Tottenham Court Road, on Tuesday, September 4, at 7.30 p.m.

The Tootal Broadhurst Lee Company has decided to set aside Io,oool. a year for five years for the promotion of research and education. According to the Times the provisional committee on research and education for the cotton industry will, at the close of the current holiday season, issue a prospectus of the new organisation. This definite industrial research federation of the cotton trade will be followed by the establishment of institutes and laboratories. It is also stated that a provisional committee to organise textile research associations in the woollen trade has been formed.

THE President of the Board of Education has appointed a departmental committee to inquire into the principles which should determine the fixing of salaries for teachers in secondary and technical schools, schools of art, training colleges, and other institutions for higher education (other than university institutions), due regard being had to such differentiation in respect of locality, duties, qualifications, sex, and other relevant considerations as is consistent with or necessary for the organisation of the teaching service throughout the country on a system conducive to the efficiency of national education. The committee is not asked to consider the question of the amounts by which existing salaries should be improved in particular areas or schools, or the sources from which the amounts required for that purpose should be provided.

We have received from the British Esperanto Association, I7 Hart Street, W.C.I, an interesting pamphlet br Mr. Bernard Long, entitled "Esperanto and Why We Need It." Mr. Long considers that whatever relations may become necessary or desirable with our present enemies after the war, it would be better to use a neutral language, whenever possible, than to accentuate existing differences by employing any of the nationai tongues, with their attendant "atmosphere" 